\title{
EVALUACIÓN DE UN PLAN INSTRUCCIONAL DIRIGIDO HACIA LA EVOLUCIÓN DE LAS CONCEPCIONES DE LOS ESTUDIANTES ACERCA DE CIRCUITOS ELÉCTRICOS
}

\author{
ANDRÉS, M.M. \\ Liceo «Rafael Seijas». Caracas.
}

\begin{abstract}
SUMMARY
This paper presents the results of a research with high school students. The work began with the diagnosis of conceptions in the students about the electrics circuit. Then it evaluated the effect of the instructional proposition. The instruction intended to stimulate the conceptual conflict and produce the conceptual change in the students.
\end{abstract}

\section{INTRODUCCIÓN}

La enseñanza de los contenidos: corriente eléctrica, relación entre diferencia de potencial y la intensidad de corriente, ley de Ohm y comportamiento eléctrico de los circuitos simples, parece estar centrada en aspectos algorítmicos; es decir, los estudiantes aprenden a manipular reglas y ecuaciones para resolver problemas cuantitativos. Los estudiantes aprueban cursos de electricidad resolviendo exitosamente problemas mediante la aplicación de leyes de Kirchoff, tey de Ohm y otros, sin embargo no desarrollan una estructura conceptual coherente con las teorías científicas, ya que al presentarles situaciones cualitativas responden erróneamente (Fredette y Lochhead 1980, Cohen et al. 1983, Criscuolo 1984, Shipstone 1988).

Las respuestas erróneas de los estudiantes no parecen ser producto de la instrucción. Antes de ingresar en los cursos de electricidad ya tienen esquemas conceptuales acerca de los circuitos y la corriente eléctrica. En diversos estudios realizados en el área de electricidad se han detectado ciertas regularidades en los patrones de razonamiento empleados por los estudiantes antes de la instrucción, especialmente en lo referente a circuitos eléctricos, corriente, voltaje, conservación de la corriente eléctrica (Evans 1978, Fredette y Lochhead 1980 , Cohen et al, 1983, Shipstone 1985, Domínguez y Moreira 1987). Estos esquemas persisten en sus estructuras conceptuales después de la instrucción (Domínguez y Moreira 1987, Criscuolo 1984).

La instrucción tal como se ha desarrollado hasta el momento parece ser poco exitosa en el desarrollo de una comprensión de los conceptos del área de electricidad. Los estudiantes adquieren conocimientos de tipo operativo, reglas y algoritmos, que les permiten resolver problemas cuantitativos. Este conocimiento no está asociado a los conceptos científicos respectivos, por lo cual ellos no podrán valorar correctamente sus resultados numéricos, pues sus esquemas conceptuales de conocimiento declarativo contienen patrones muy particulares.

La instrucción debe lograr que el estudiante conozca sus patrones de razonamiento, y ponerlos a prueba en diversas situaciones. El docente debe diseñar o seleccionar los eventos instruccionales que permitan poner en evidencia la incoherencia de dichos patrones y orienten al 
estudiante hacia el desarrollo de estructuras cognoscitivas integradas por conocimientos científicamente aceptados. En el caso de Física y en particular en el área de interés de este trabajo: la electricidad, la instrucción debe proporcionar al estudiante situaciones de análisis cualitativo y posteriormente eventos donde se relacione esto con las operaciones cuantitativas (Criscuolo 1984).

La propuesta instruccional, denominada para efectos de este trabajo "estrategia de conflicto», enfatiza la comprensión conceptual y toma en cuenta las ideas de los estudiantes. Está fundamentada en los planteamientos de Posner (1982), quien considera la construcción del conocimiento como un proceso evolutivo en el cual los cambios se producen a medida que las nuevas ideas se adecúan mejor a la situación real y permiten explicar mayor número de eventos. La estrategia de conflicto intenta poner en evidencia las limitaciones de las concepciones de los estudiantes, que ellos sientan la necesidad de cambiarlas o modificarlas

La secuencia de los contenidos a enseñar fue la siguiente: diferencia de potencial, fuerza electromotriz de las baterías, corriente eléctrica, resistencia, conductividad, relación entre la diferencia de potencial y la intensidad de corriente en elementos lineales y no lineales, ley de $\mathrm{Ohm}$, análisis cualitativo de los circuitos simples y posterior análisis cuantitativo.

La selección de los eventos es de suma importancia ya que de ello depende la externalización de las concepciones de los estudiantes y el surgimiento del conflicto. La estrategia de conflicto presenta cuatro fases: predicción, observación, discusión reflexiva y generación del conocimiento.

La fase de predicción: a los alumnos se les provee de una descripción del evento y se le pide que hagan sus predicciones acerca de lo que sucederá, así como la explicación que las sustenta.

La fase de observación: los estudiantes diseñarán las experiencias necesarias para verificar sus predicciones, por lo tanto recogerán las observaciones necesarias .

La fase de discusión reflexiva: se discutirá acerca de la validez de sus explicaciones, a raíz de lo cual pueden surgir nuevas experiencias.

La fase de generación del conocimiento: se hará la modificación de sus explicaciones iniciales o el cambio de ellas en función de los resultados y de los modelos científicos.

\section{OBJETIVOS DEL TRABAJO}

1. Evaluar el diseño instruccional propuesto para el logro de la evolución conceptual de los patrones de conocimiento de los estudiantes hacia los modelos científicos.
2. Determinar el efecto del curso de electricidad de escuela básica sobre las concepciones de los estudiantes antes y después de la instrucción experimental.

3. Relacionar el sexo con la puntuación en la prueba de concepciones acerca de electricidad (DCE) antes y después de la instrucción.

4. Determinar la correlación entre rendimiento en Física, rendimiento en la prueba final de lapso, antes y después de prueba DCE.

\section{METODOLOGÍA}

En Venezuela la educación básica (EB) comprende 9 años y la educación diversificada comprende 2 ó 3 años, según la opción. En los tres últimos años đe $\mathrm{EB}$ algunos estudiantes tienen la posibilidad de cursar electricidad (curso de formación para el trabajo, de carácter técnico). La asignatura de Física se cursa formalmente en el noveno grado de EB. Si toma la opción de Ciencias cursará Física en los dos años de eđucación diversificada. Los contenidos de electricidad aparecen en el programa del último curso de Física

El trabajo fue una investigación de tipo preexperimental: un grupo experimental con pre y postprueba (Campbell 1973). La muestra fue de tipo accidental, conformada por dos cursos del último año de la educación secundaria para un total de 73 alumnos. La edad promedio era de 17 años. En cuanto al sexo, había 51 varones y 22 hembras. En la muestra había 36 alumnos que durante los tres últimos años de EB cursaron electricidad.

\section{INSTRUMENTOS DE MEDICIÓN}

El rendimiento en la prueba tradicional (PFL) se midió a través del examen final del trimestre, el cual evaluaba los aprendizajes logrados por los alumnos durante el lapso. Esta prueba combina preguntas cuantitativas con preguntas cualitativas y, preguntas de ensayo con preguntas de selección simple.

La prueba para determinar las concepciones de los estudiantes fue diseñada por el autor, para ello se emplearon preguntas de otros trabajos afines o la adaptación de ellas. La prueba fue validada por el juicio de expertos. Los contenidos evaluados en la prueba de determinación de Concepciones sobre electricidad (DCE) (Anexo 1) son:

Ítem 1: Circuito: un bombillo y una pila unida con cables.

1.1,1.2,1.6 -> los cables provienen de los dos polos de la pila y tocan un terminal del bombillo.

$1.5->$ los cables provienen de un solo polo de la pila y tocan un solo terminal del bombillo. 


\section{3,1.4 -> los cables hacen contacto con los 2 polos de la} pila y los 2 terminales del bombillo.

Ítem 2: Corriente eléctrica.

Ítem 3: Aditividad del potencial

$3.1->$ dos pilas de $1.5 \mathrm{~V}$. en serie

$3.2->$ dos pilas de $1.5 \mathrm{~V}$. en paralelo.

Ítem 4: Circuito en serie.

Ítem 5: Circuito en paralelo.

Ítem 6: Comparación de la serie con el paralelo.

Ítem 7: Discriminación entre voltaje y potencia.

Ítem 8: Caso de un bombillo que se quema.

El rendimiento en Física se determinó por el promedio de calificaciones aprobatorias obtenidas en los cursos de Física de $9^{\circ}$ de educación básica y $1^{\circ}$ de la educación diversificada.

\section{PLAN DE INSTRUCCIÓN PROPUESTO}

Los estudiantes tuvieron un rol activo dentro de este plan, eran los protagonistas principales de las actividades de aprendizaje propuestas, las cuales variaban: discusión de lecturas, simulaciones, actividades de laboratorio, elaboración y discusión de reportes de laboratorio y sesiones de solución de problemas.

El diseño instruccional tuvo una duración de 30 clases de 40 minutos, agrupadas en bloques de 2 clases y distribuidas en 8 semanas. Las sesiones estaban programadas para trabajar con todo el curso (36) o con la mitad, (18) según las necesidades. Las sesiones de clase estaban dirigidas mediantes hojas de trabajo en las cuales se les indicaban los objetivos y las actividades a realizar. En las actividades de laboratorio se apitcó la estrategia de conflicto. Las hojas de trabajo contenían los objetivos y las descripciones de los eventos, acompañadas de preguntas que activasen los esquemas de los estudiantes y generasen nuevas preguntas.

\section{EQUIPO DE LABORATORIO}

Los recursos de laboratorio requeridos en este plan instruccional son: un tablero eléctrico para cada tres estudiantes, cables de teléfono de $\cong 20 \mathrm{~cm}$ de longitud, pilas de $1.5 \mathrm{~V}$., bombillo de rosca de $1.5 \mathrm{~V}$. y $6 \mathrm{~V}$., porta bombillos con sus cables de conexión, resistencias, amperímetro y voltímetro.

\section{RESULTADOS Y ANÁLISIS}

Análisis cualitativo de los resultados obtenidos con la prueba DCE, antes y después de la instrucción experimental.

A continuación se expondrán los resultados de la aplicación de la prueba DCE en términos de porcentajes de respuestas: correctas, incorrectas, confusas o correctas con explicaciones incompletas y no respondidas. (Tabla I, Figs. 1-2)

\section{Tabla I}

Resultados de la prueba determinación de concepciones acerca de electricidad (DCE) antes y después de la experiencia.

(Porcentaje de respuestas)

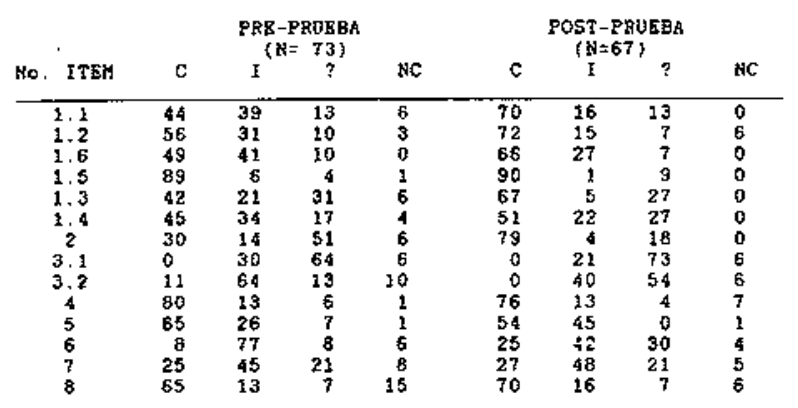

$\mathrm{c}=\operatorname{correcta}$

$1=$ incorrecte

$?=$ confusa
$\mathrm{NC}=$ no contesto

En función de estos resultados, se mostrarán los patrones de concepciones de los estudiantes antes y después de la instrucción experimental:

A. El funcionamiento de un circuito es explicado por los estudiantes de la siguiente manera:

«Un circuito debe estar alimentado por los dos tipos de carga de la fuente, + y ; estas cargas fluyen por los cables pasando por el bombillo".

Antes de la instrucción muchos estudiantes pensaban que lo importante era que al bombillo entraran o llegasen los dos tipos de carga. Después de la instrucción el patrón de modifica: "Las cargas deben entrar por un terminal del bombillo y salir por el otro, juntándose en el filamento los dos tipos de cargam.

Después de la instrucción parece que el concepto de círcuito está más claro, conocen cómo está constituido un bombillo y su funcionamiento, pero pese a que definen la corriente eléctrica como el flujo de electrones a través de un conductor hablan de que llegan los dos tipos de cargas al bombillo. 
figuras 1 y 2

RESULTADOS PRE Y POST PRUEBA

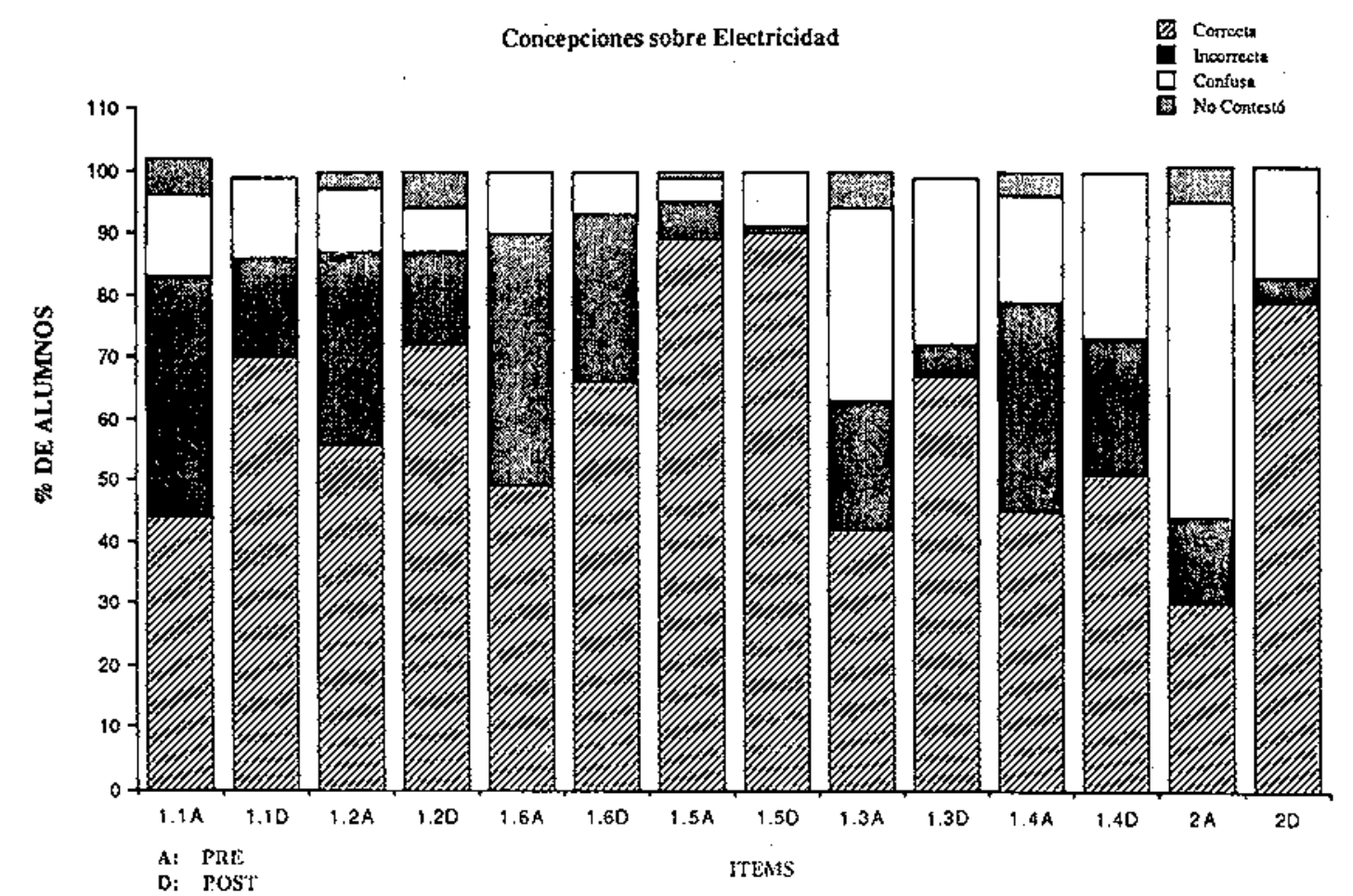

RESULTADOS PRE Y POST PRUEBA
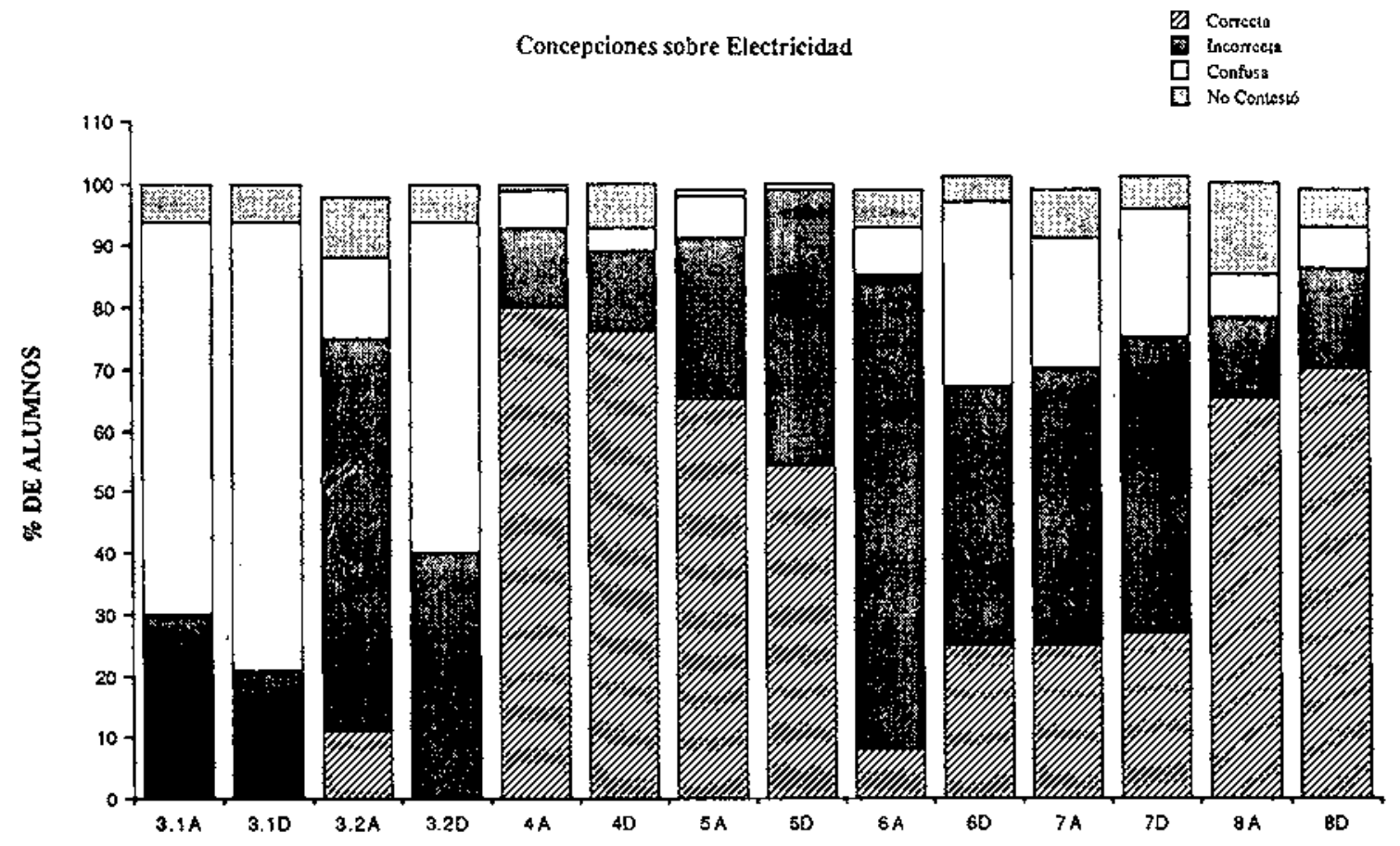
B. En lo referente a los circuitos en serie y paralelo los patrones más comunes son:

Serie: «Llega (energía, voltaje, cargas) a ambos bombillos de manera simultánea y en igual proporción a los dos, descle los polos de la fuente; el cable del medio permite que éstas puedan circular».

Paralelo: «Llega (carga, energía, electricidad, corriente) al bombillo más cercano a la fuente, éste absorbe (consume) la mayor parte y el resto lo pasa al bombillo siguiente, por eso ilumina más el bombillo cercano a la fuente».

«Brillan igual porque a los bombilios les llega la misma cantidad (carga, energía, electricidad, corriente)».

Al comparar la luminosidad de los bombillos en paralelo con los bombillos en serie se encontró:

«Brillan igual en los dos circuitos, ya que el brillo depende de la fuente y tipo de bombillo, si éstos son idénticos y usamos la misma fuente no influye la posición de los elementos del circuito, los bombillos tendrán igual iuminosidad».

«En serie brillan más que en paralelo; en la serie, a los bombillos les llega la misma cantidad de carga de manera directa y simultánea, mientras que en el paralelo un solo bombillo está unido a la fuente de forma directa».

C. La instrucción no influyó en los patrones de concepciones de los estudiantes referentes a la asociación de pilas. El patrón más frecuente para la serie es:

«3 V., por estar polos opuestos unidos haciendo que las cargas se atraigan o se unan».

Los patrones más comunes para la asociación de pilas en paralelo son:

$« 0 \mathrm{~V}$. cargas iguales se repelen, por lo tanto se anulan entre sí, restándose los voitajes».

«1.5 V., las cargas del mismo tipo no se suman».

\section{Análisis estadístico}

A. El efecto del curso de electricidad de EB sobre el resultado en la prueba DCE se analizó con la prueba U de Mann-Whitney (Siegel 1980). Las hipótesis propuestas fueron:

$\mathrm{H}_{1}$ : Los alumnos que no cursaron la asignatura de electricidad en EB obtendrán menor puntuación en la prueba DCE que aquéllos que sí la cursaron.

$\mathrm{H}_{0}$ : Los alumnos que no cursaron la asignatura de elec. tricidad en EB obtendrán iguales puntuaciones en la prueba DCE que aquéllos que sí la cursaron.

Los resultados antes de la instrucción dieron un $\mathrm{z}=1.43$, por lo tanto no se rechaza la hipótesis nula. El haber cursado electricidad en EB no influyó en los resultados de la prueba DCE. Sin embargo, después de la instruc* ción se obtuvo un $z=2.50$, el cual es significativo para un 0.01, rechazándose en este caso la hipótesis nula. Los estudiantes que cursaron electricidad en $\mathrm{EB}$ obtuvieron mayores puntuaciones en la prueba DCE aplicada como postest que aquéllos que no cursaron electricidad en EB.

B. La diferencia entre los resultados en la prueba DCE según el sexo fue analizada mediante la prueba $U$ de Mann-Whitney. Las hipótesis planteadas fueron:

$\mathrm{H}_{1}$ : Los varones tendrán mayores puntuaciones en la prueba DCE que las hembras.

$\mathrm{H}_{0}$ : Las puntuaciones obtenidos en la prueba DCE no diferirán entre varones y hembras.

Los resultados de la prueba DCE aplicada antes de la instrucción arrojó un $z=2.15$, el cual es significativo para un 0.02 , por lo tanto se rechaza la hipótesis nula. Después de la experiencia la diferencia se hace menos marcada; se obtuvo un $\mathrm{z}=1.78$, el cual es significativo para un 0.04 , rechazándose la hipótesis nula.

C. Se analizó la correlacion entre las variables: Rendimiento en Física (RF), prueba final de lapso (PFL), y pre y postprueba DCE. Se analizaron la correlaciones mediante el coeficiente comelación de Speaman $\left(\mathrm{r}_{s}\right)$ (Siegel 1980).

Según la tabla II se puede ver que la correlación entre RF y preprueba DCE no es significativa, en cambio para el caso de RF y postprueba DCE el coeficiente es de $\mathrm{r}_{\mathrm{s}}$ : 0.24 , el cual es significativo para un 0.025 . Entre la PFL, y la pre y postprueba DCE se encontraron coeficientes significativos $\left(r_{\mathrm{s}}: 0.2, p=0.05\right.$ con el pretest; $r_{\mathrm{s}}: 0.40$, $p=0.0005$ con el post) Aquí nuevamente hubo mayor correlación con el postest que con el pretest. Se observa también que entre la preprueba y el postprueba DCE la correlación es baja y no significativa.

Tabia II

Cuadro de cortelaciones entre las variables : rendimiento en física (RF). Prueba final de lapso (PFL). Pre y post de la prueba de determinacion de concepciones acerca de electricidad (DCE).

\begin{tabular}{lllll} 
& RF & PFL & PRE-DCE & POST-DCE \\
\hline PFL & $\frac{1}{0.47^{*}}$ & 1 & \\
\hline PRE-DCE & $0.18^{*}$ & $0.20^{*}$ & \\
\hline POST-DCE & $0.21^{*}$ & $0.40^{* *}$ & 0.18 & 1 \\
\hline
\end{tabular}

* $\quad 0.05$ 


\section{CONCLUSIONES}

El curso de electricidad realizado en los tres últimos años de EB no influyó en la manera de responder a la prueba DCE aplicada como pretest. Pudiera pensarse en el papel que juega el tiempo dentro de la memoria de los estudiantes, en ese momento hacía más de un año que no trabajaban con el área de electricidad, las preguntas de la prueba DCE no mostraron ningún conocimiento escolar, ellos utilizaron sus esquemas particulares para responder a las preguntas de la prueba. Por otra parte, se observa que el aprendizaje logrado en dichos cursos fue temporal ya que después de un año responden como aquellos estudiantes que nunca cursaron dicha asignatura.

La experiencia instruccional tuvo mayor éxito en los estudiantes que habían tenido la vivencia del curso de electricidad. Parece que el experimento les activó los esquemas adquiridos en dichos cursos, permitiéndoles mayor comprensión de los contenidos y de los fenómenos.

Las diferencias encontradas entre los varones y las hembras en cuanto a los resultados en la prueba DCE pudiera tener explicación desde el punto de vista cultural y social. Existe una diferenciación entre el rol y las actividades de la mujer y del hombre. Aun cuando la incursión de la mujer en el mundo tecnológico y científico cada vez es más alta, continúa existiendo la creencia de que áreas como la electricidad son más propias del sexo masculino. Este es un aspecto que debería ser estudiado; conocer las creencias de los estudiantes al respecto permitiría poder romper con las limitaciones culturales dentro del aprendizaje de los temas sobre electricidad.

Los estudiantes modificaron sus concepciones iniciales en lo referente a circuito y corriente eléctrica. Inicialmente empleaban el modelo de choques de corriente para luego pasar al uso del modelo científico, lo cual coincide con los resultados de Shipstone (1984).

En cuanto al circuito en serie, se encontró que los alumnos no empleaban el modelo de atenuación (Criscuolo 1984, Dominguez y Moreira 1987). Sin embargo, la explicación que dan a la igualdad de la iluminación de los bombillos muestra que no discriminan entre los conceptos de energía, voltaje y corriente, to cual se mantiene después de la instrucción.

En el circuito en paralelo un porcentaje de estudiantes considera que la cercanía del bombillo a la fuente es factor determinante en su iluminación, lo cual no se modifica con la instrucción. Entre aquéllos que consideran que tendrán igual luminosidad se encuentra que no discriminan entre energía, voltaje, y corriente.

La propuesta para próximos trabajos está centrada en mantener la estrategia de conflicto pero aumentando la cantidad de eventos. Por ejemplo, trabajar la serie con 2 , 3 y más bombillos, luego realizar experiencias con 2,3 y más resistencias midiendo en estos últimos la intensidad de la corriente y la diferencía de potencial con los amperímetros y voltímetros. La fase de generación de conocimientos estaría sustentada por mayor número de evidencias acerca de la validez de las conclusiones (esquemas científicos).

La instrucción debe hacer mayor énfasis en el funcionamiento interno de las fuentes generadoras de energía empleadas en los circuitos. Se considera necesario la discriminación entre la f.e.m. y la diferencia de potencial $\mathrm{y}$ al mismo tiempo su relación. Igualmente se considera necesario discutir la relación entre la energía de la fuente, la f.e.m. y la $\Delta \mathrm{V}$ en los terminales de la fuente conectada al circuito.

El rol del laboratorio es fundamental en el proceso instruccional de este tema. Las demostraciones orales o escritas mediante el lenguaje de la matemática sin el empleo de la estrategia de conflicto logra el aprendizaje memorístico de reglas, por el principio de la autoridad, no se alcanza la comprensión de los conceptos; tal como ocurrió en esta experiencia con la asociación de pilas en serie y paralelo (ver resultados de los ítems 3.1 y 3.2).

La comprensión conceptual y el cambio conceptual exigen la inversión de tiempo en la instrucción. En el diseño del currículo se debe tener claro el objetivo a lograr. Si se desea que los alumnos adquieran información, la cantidad de contenidos puede ser mayor pero a costa de una menor comprensión conceptual. Por el contrario si se desea la comprensión conceptual se debe disminuir la cantidad de contenidos para ampliar el número de las actividades instruccionales por cada uno de ellos.

La búsqueda de propuestas instruccionales, de activida* des de aprendizaje dirigidas al logro de la comprensión conceptual y del cambio conceptual debe continuar. Es necesario lograr que los estudiantes sean capaces de elaborar las repuestas a las preguntas planteadas mediante la activación de los esquemas conceptuales adecuados. Hay que erradicar la conducta mecanizada, las respuestas estandarizadas por problemas tipos. 


\section{ANEXO I}

Prueba de Física. Concepciones sobre tópicos de electricidad. (Resumen de las cuestiones planteadas. No se reproducen los esquemas.)

1. Si te damos un bombillo, dos cables y una pila, y te pedimos que los unas de forma que el bombillo dé huz. ¿Cuál(es) de los diagramas representa una conexión adecuada? (Se incluyen seis esquemas.)

Explica en cada caso por qué el bombillo da o no da luz.

2. Al tocar un cable de luz que perdió la cubierta de plástico, es decir pelado, decimos que nos dio "corriente". ¿Qué significa para ì el término corriente?

3. Se tienen dos pilas de 1,5 Voltios; ellas pueden conectarse entre sí de dos maneras. ¿Cuál será el voltaje que obtenemos en cada conexion? Explica tu respuesta.

4. Tienes dos bombillos iguales ( 10 vatios) conectados a una batería tal como la figura (en serie). ¿Cómo será el brillo de los bombillos?

Igual. Mayor en 1 que en 2. Mayor en 2 que en 1 . Razonamiento.

5. Los bombillos anteriores se conectan a la misma batería pero como lo señala la figura (en paralelo). ¿Cómo será el brillo de los bombillos?

Igual. Mayor en 1 que en 2. Mayor en 2 que en 1. Razonamiento.

6. Si comparas el brillo de los bombillos de la conexión del ítem 4 con los de la conexión del ítem 5 , ¿Como será el brillo de los bombillos?

Igual en 4 y en 5 . Mayor en 4 que en 5. Mayor en 5 que en 4. Razonamiento.

\section{REFERENCIAS BIBLIOGRÁFICAS}

ANDRÉS, M., 1982. Diseño instruccional para el aprendizaje de los conceptos de corriente eléctrica $e$ intensidad de corriente. Trabajo sin publicar realizado para la cátedra de Diseño de instrucción.

CAMPBELL, D. y STANLEY, J., 1973. Diseños experimentales y cuasiexperimentales en la investigación social. (Amorrortu).

COHEN, R., EYLON, B. y GANIEL, U., 1983. Potential difference and current in simple electric circuits: a study of student's concepts, American Journal of Physics, 50(5), pp. $407-412$.

CRISCUOLO, G., 1984. Concepciones espontáneas sobre la circulación de corriente en circuitos de corriente continua. II Jornada sobre enseñanza de la Física CENAMEC. Caracas.

DOMINGUEZ, E. y MOREIRA, M.A., 1987. Stability of misconceptions on electric current among college students. I Conferencia Interamericana de enseñanza de la Física. México.
EVANS, J., 1978. Teaching electricity with batteries and bulbs, the Physics Teacher, 16(1), pp. 15-24.

FREDETTE, N, y LOCHHEAD, J., 1980. Student conceptions of simple circuits, The Physics Teacher, 18(3), pp. 194-198.

POSNER, G., 1983. A model of conceptual change: present status and prospect, en Novak, J.y Helm, H. (eds.), Proceedings of the misconceptions in science and mathematics. (Univ. Comeil Ithaca: N.Y.), pp. 71-75.

SEBASTIÀ, J., 1988. La comprensión de los circuitos eléc. tricos elementales. Seminario permanente de la enseñanza de Ia Fisica, U.S.B. Jun-88.

SIEGEL, S., 1980. Estadística no paramétrica. (Trillas: México).

SHIPSTONE, D., 1985. Electricity in simple circuits, en Driver, R. et al. (eds.), Children's ideas in science. (Milton Keynes: Open University Press).

SHIPSTONE, D., 1988. Pupil's understanding of simple electrical circuits: some implications for instruction, Physics Education, vol. 23, pp. 92-96. 